\title{
Evaluación del dolor musculoesquelético en el anciano
}

\author{
Assessment of musculoskeletal pain in the \\ elderly
}

\author{
Carlos Ernesto Arteaga, Juan Guillermo Santacruz, \\ LIZETH Jazmín Ramírez • Bogotá, D.C.
}

\section{Resumen}

Propósito: las afecciones musculoesqueléticas son causa frecuente de dolor y discapacidad en el anciano, el conocimiento de estas patologías se hace necesario dado que la presentación de algunas de ellas son diferente en los adultos mayores, tales como artritis reumatoide, lupus eritematoso sistémico, fibromialgia, y otras patologías son más frecuentes entre ellos, así como la osteartritis y polimialgia reumática. Por ende el abordaje diagnóstico y terapéutico es de vital importancia dado los cambios que se presentan en este grupo poblacional secundarios al envejecimiento.

Fuente de obtención de los datos: se realizó una búsqueda de literatura a través de pubmed, considerando epidemiologia, enfoque diagnóstico y terapéutico de las afecciones musculoesqueléticas en el adulto mayor, enfocando actualizaciones en temas de revisión.

Síntesis de los datos: el dolor musculoesquelético tiene un bajo abordaje diagnóstico y terapéutico debido a que la mayoría del personal de salud lo considera como secundario al envejecimiento. Se hace una revisión de las principales patologías musculoesqueléticas y las diferencias en el manejo terapéutico a considerar en el anciano.

Conclusiones: el dolor musculoesquelético es una causa frecuente discapacidad en el anciano, su correcto diagnóstico hace que se reduzcan secuelas funcionales dadas por la inmovilidad secundaria y aislamiento social que conlleva. Igualmente debe considerarse que el envejecimiento puede afectar la farmacodinamia, por lo cual algunos fármacos deben usarse con cautela en los adultos mayores (Acta Med Colomb 2011; 36: 30-35).

Palabras clave: enfermedades reumáticas, ancianos, dolor, discapacidad, farmacología.

\section{Abstract}

Purpose: musculoskeletal disorders are common causes of pain and disability in the elderly. Knowledge of these conditions is necessary, since the clinical presentation of some of them is different in older adults. Among these illnesses are rheumatoid arthritis, systemic lupus erythematosus, fibromyalgia, osteoarthritis, and polymyalgia rheumatica. The diagnostic and therapeutic approach is vital in view of the changes that occur in this population due to aging.

Source of data collection: a systematic review of the literature through PubMed, searching for epidemiology, diagnosis and therapeutic approach of musculoskeletal disorders in the elderly; only topic revisions were chosen.

Data Synthesis: musculoskeletal pain has an unsatisfactory diagnostic and therapeutic approach, because the majority of health workers consider it to be due to aging. We review major musculoskeletal conditions and differences in the therapeutic management to be considered in the elderly.

Conclusions: musculoskeletal pain is a common cause of disability in the elderly. Correct diagnosis reduces its functional consequences, such as immobility and social isolation. It must also be considered that aging can affect drug kinetics, so that some drugs should be used cautiously in older adults (Acta Med Colomb 2011; 36: 30-35).

Keywords: rheumatic diseases, elderly, pain, disability, pharmacology
Dr. Carlos Ernesto Arteaga: Internista, Hospital Universitario Mayor de Cundinamarca- Mederi. Especialista en Medicina Interna, Universidad El Bosque - Fundación Santa Fe de Bogotá. Médico y Cirujano, Universidad de Caldas; Dr. Juan Guillermo Santacruz: Coordinador del Servicio de Cuidado Paliativo Fundación Santa Fe de Bogotá. Subespecialista en Dolor y Cuidado Paliativo, Universidad Pontificia Javeriana. Especialista en Medicina Interna, Médico y Cirujano, Pontificia Universidad Javeriana; Dra. Lizeth Jazmín Ramírez Bustamante: Fellow de Dolor y Cuidado Paliativo, Universidad El Bosque, Bogotá, D.C. Especialista en Anestesiología, Universidad de Caldas. Médico y Cirujano, Universidad Pontificia Bolivariana.

Institución donde fue realizado el trabajo: Fundación Santa Fe de Bogotá.

Presentación a congresos: no

Patrocinio financiero: no

Correspondencia: Dr. Carlos Arteaga Unigarro

E-mail: cauniga@gmail.com

Recibido: 04/VIII/2010 Aceptado: 17/I/2011 
Debido al aumento en la tasa de envejecimiento a nivel mundial, es necesario ampliar el conocimiento en el manejo de algunas patologías, las enfermedades discapacitantes crónicas por ejemplo, tienen mayor frecuencia en el anciano; siendo el dolor musculoesquelético en muchas ocasiones causa de esta discapacidad, existen patologías como la polimialgía reumática, la osteartritis que son más comunes en ancianos.

El dolor musculoesquelético en el adulto mayor es una de las quejas más frecuente, cerca del 25-80\% de los ancianos en algún momento presenta algún tipo de dolor (1); en ancianos institucionalizados llega al 45-80\% vs los pacientes que viene con sus familias que sólo lo presentan del 25-50\%, reflejado el componente sicosomático del dolor. Pese a esta gran prevalencia, la mayoría de médicos subestima la importancia de evaluación y manejo de dolor en los pacientes de edad avanzada y lo consideran parte natural del envejecimiento, sin recordar que el dolor puede influir en el estado de ánimo, el funcionamiento físico y las interacciones sociales, indicando que el abordaje y manejo es multidimensional y multidisciplinario (2). La pérdida de acondicionamiento muscular y las alteraciones de la marcha secundarias a dolor, pueden dar lugar a lesiones por caídas, alteraciones del apetito, y del sueño (3), situación que lleva a menor calidad de vida y mayores costos en salud.

\section{Abordaje del dolor en el anciano}

La evaluación del dolor en el anciano, no es tan diferente a la del adulto joven, se trata de identificar la causa del dolor, realizando una historia completa que incluya comorbilidad médica psicosocial y una exploración física completa. El autoinforme de dolor se considera la fuente más fiable para los ancianos con capacidad cognitiva intacta, pero debe tenerse en cuente las limitaciones que pueden tener; alteraciones auditivas, visuales, cognitivas y sensoriales que pueden interferir al momento de la evaluación, por ello es de utilidad la entrevista con miembros de la familia o cuidadores para poder caracterizar mejor el síntoma y la repercusión en el estado de ánimo, el afecto y la funcionalidad física y social (4-8), en los ancianos con deterioro cognitivo, la evaluación se centra en cambios del comportamiento secundarios y señales no verbales, vocalizaciones y expresiones faciales (9-11).

\section{Causas de dolor musculoesquelético en el adulto mayor}

Las causas de dolor musculoesquelético en el anciano son múltiples, ya sean enfermedad crónica iniciada en la juventud o problemas reumatológicos que son más frecuentes a esta edad. Los siguientes son algunas de las patologías por considerar:

\section{Dolor lumbar}

Cerca del $30 \%$ de los adultos mayores de 65 años presentan quejas de dolor lumbar, que pese a ser en muchas ocasiones causa de dolor por alteración de desacondicionamiento y secundario a contracturas musculares y retracciones, debe explorarse al presentarse o agudizarse porque hay entidades como ruptura de aneurisma de aorta abdominal, disección aortica, síndrome de compresión medular, fractura vertebral, que ponen en riesgo la vida o causan severa discapacidad (12).

\section{Osteoartritis (OA)}

La prevalencia de la OA en la los ancianos es más alta que en pacientes de menor edad, afecta con mayor frecuencia a mujeres, con mayor incidencia entre los 65 y 74 años, aproximadamente 13.5 casos por 1000 personas al año, en hombres la mayor incidencia está en mayores de 75 años y es de aproximadamente nueve casos por 1000 personas al año. Se estima que el $9.6 \%$ de los hombres y el $18 \%$ de las mujeres mayores de 60 años tienen OA sintomática. La OA de rodillas es la principal causa de deterioro en la movilidad, especialmente en mujeres, responsable del $2.8 \%$ del total de años vividos con discapacidad (10-13). Asociado a la debilidad del cuadriceps que es un factor predictor que aumenta el grado de discapacidad y postración $(14,15)$.

\section{Artiris reumatoide (AR)}

La AR es la artropatía inflamatoria más frecuente, con prevalencia de $0.3-1 \%$, y mucho más frecuente entre los ancianos (16). Existe además una forma de presentación en el anciano que se considera de inicio tardío, un tercio de los pacientes debuta después de los 60 años. La relación mujerhombre en la AR es 3:1, en la forma tardía la afectación es por igual 1:1 (17-18) más aguda, de articulaciones proximales, acompañado de manifestaciones sistémicas; como fatiga, pérdida de peso, VSG elevada. El factor reumatoide es positivo sólo en $32-58 \%$, pero al estar presente la enfermedad es más severa, acompañada de depresión, y deterioro en la calidad de vida (19), con mayor frecuencia en fractura de cadera (20). Debe diferenciarse la AR de inicio tardío de otros trastornos reumatológicos más frecuentes en esta población, como la polimialgia reumática, artritis inducida por cristales u osteoartritis, del diagnóstico precoz de la AR depende el tratamiento temprano para lograr la remisión clínica o el menor nivel posible de actividad de la enfermedad para minimizar las secuelas funcionales. Algunos casos la AR de inicio tardío con factor reumatoide negativo y síntomas similares a la polimialgia reumática parece ser un subconjunto distinto, con base genética diferente y curso más benigno. El tratamiento prudente también es necesario por el riesgo de eventos adversos en pacientes de edad avanzada. Sin embargo, no existe ninguna contraindicación especial para la utilización de fármacos antirreumáticos modificadores de enfermedad en este grupo de edad, ni del uso en terapia biológica.

\section{Reumatismo de tejidos blandos}

El término reumatismo de tejidos blandos es una de las múltiples designaciones de un grupo de entidades que se 
caracterizan por dolor regional de las extremidades y cuya causa habitual es lesión de tendones, entesis y bursas. La mayoría de las veces el dolor del eje axial es secundario a patología mecánica, pero en este grupo de edad son más frecuentes las fracturas vertebrales osteoporóticas, el mieloma múltiple o las metástasis óseas. La secuencia terapéutica habitual en dolor mecánico comprende inicialmente la supresión o modificación de los factores causales, el uso de antiinflamatorios, la fisioterapia y en casos rebeldes a estas terapias la inyección local con glucocorticoides o la cirugía (21-22).

\section{Fibromialgia}

La fibromialgia es más prevalante con la edad, la prevalencia es de $2 \%$ entre los 30-39 años, 5.6\% entre los 50-59 años y 7.4\% entre los 70-79 años. Las características comunes de la fibromialgia como dolor y rigidez, cansancio físico, falta de sueño, fatiga, ansiedad y edema de tejidos blandos subjetivo con múltiples puntos sensibles a la exploración física y cefalea funcional crónica están presentes igualmente en el anciano, aunque el agravamiento de los síntomas por factores ambientales, el estrés mental, y por falta de sueño, son menos frecuentes entre los personas mayores. Es importante destacar que, la fibromialgia ha sido reconocida por los médicos tratantes en sólo el 17\% de los pacientes ancianos. Errores de diagnóstico y tratamiento inadecuado son frecuentes, con uso de corticoides en el $40 \%$ antes de la consulta de reumatología. Igualmente si la aparición es por encima de los 65 años, requiere especial atención al diagnóstico diferencial, patologías concomitantes y secundarias (23-25).

\section{Artropatías por cristales}

La gota en el adulto mayor tiene una alta prevalencia, debido a la mayor duración de la hiperuricemia, enfermedades asociadas como la hipertensión, obesidad, dislipidemia, el uso de medicamentos para tratarlas como diuréticos y ácido acetilsalicílico. Afecta más a hombres pero con un leve aumento de incidencia en mujeres después de los 65 años (26). La presentación es atípica en la mayoría de casos y el diagnóstico pasa desapercibido o se establece en forma errónea. En las mujeres de 60 años la presentación de la gota puede ser poliarticular, de inicio lento, subagudo, y con compromiso de las articulaciones interfalángicas de las manos, dando lugar a confusión con AR o exacerbación de un proceso degenerativo. El examen cuidadoso del líquido sinovial y la identificación de los cristales de urato monosódico son primordiales para el diagnóstico. Recientemente por limitaciones se considera a la ultrasonografía como herramienta equivalente del mismo. El cuadro poliarticular asimétrico con crisis en algunas articulaciones, los hallazgos radiológicos característicos, la hiperuricemia, títulos de factor reumatoide negativo o positivo a títulos bajos, orientan el diagnóstico hacia gota en anciano (27). Debe descartarse causa secundaria de hiperuricemia como enfermedad mie- loproliferativa, linfoproliferativa, falla renal, hipertensión arterial, alcoholismo. La colchicina y AINES son igualmente efectivos pero con perfil de seguridad diferente, es de recordar que no debe tratarse la hiperuricemia asintomática.

En la pseudogota (condrocalcinosis) una enfermedad por depósitos de pirofosfato cálcico, no hay alteración del metabolismo del calcio, por lo que no es necesaria ninguna dieta especial. Generalmente se ve en personas mayores de 50 años de edad. Tiene alta asociación con artrosis, hiperparatiroidismo, hemocromatosis e hipotiroidismo. Es por ésto que cuando se hace el diagnóstico de pseudogota hay que descartar estas afecciones. Radiológicamente hay calcificación de los fibrocartílagos y cartílago hialino en rodillas, muñecas, línea media del pubis, cadera y discos intervertebrales. Se recomienda aspiración articular y uso de esteroide intraarticular. En el episodio agudo es de utilidad usar un antiinflamatorio no esteroide. En los casos en que hay episodios agudos frecuentes, se beneficia del uso de colchicina $(28,29)$.

\section{Lupus eritematoso sistémico (LES)}

El LES puede al igual que la AR tener un comienzo después de los 50 años. A diferencia de lo que ocurre entre las personas jóvenes, donde la relación mujer:hombre es de 5-8:1, en el adulto mayor ésta es de 2:1 y parece que se afecta más la población de raza blanca, la presentación es insidiosa con menos compromiso de órgano, presentando síntomas constitucionales, artralgias, mialgias y linfoadenopatías, menor actividad. Debe diferenciarlo de otras entidades reumatológicas (polimialgia reumática, artritis reumatoide de inicio tardío, síndrome de Sjögren) y no reumatológicas (neoplasias, endocrinopatías), es frecuente la serositis, enfermedad pulmonar intersticial, síndrome de Sjögren. En cuanto a los anticuerpos antinucleares (ANAs) han sido reportadas en un $14 \%$ de los adultos mayores sin enfermedad autoinmune, pueden ser positivos en otras enfermedades como artritis reumatoide, hepatitis autoinmune, neoplasias, algunos medicamentos pueden inducir ANAs positivos (mayor riesgo dado la polifarmacia), o cuadros de LES inducido por medicamento, para lo cual los anticuerpos anticromatinas al estar presentes confirman este diagnóstico. Por ello los ANAs deben estar en títulos altos para ser más específicos. Distintos estudios han encontrado una alta frecuencia de anti-Ro/SSA, anti-La/SSB y de factor reumatoide en pacientes con LES tardío, con baja presencia de anti-Sm y anti-DNA de cadena doble (30-31). A pesar de tener menor compromiso renal y actividad de la enfermedad hay factores que podrían ensombrecer su pronóstico como la presencia de compromiso pulmonar, el envejecimiento en sí, mayor número de comorbilidades que impactaran negativamente su pronóstico.

\section{Polimialgia reumática}

La polimialgia reumática es una enfermedad inflamatoria que afecta generalmente a personas mayores de 50 años, se 
caracteriza por dolor y debilidad de músculos proximales de más de un mes de evolucion, junto con elevación de la velocidad de sedimentación globular (VSG) superior a 40 $\mathrm{mm} / \mathrm{h}$ (es infrecuente que sea entre 80 y $100 \mathrm{~mm} / \mathrm{h}$ ), aunque hasta un 20\% pueden tener VSG normal, tiene una relación hombre: mujeres 1:3, es frecuente encontrar anemia normocítica, normocrómica hasta en un $20 \%$, se puede asociar con arteritis de células gigantes. La respuesta a corticoides es excelente y hace diagnóstico (32). Se recomienda iniciar prednisolona $15 \mathrm{mg} / \mathrm{d}$ durante tres semanas, disminuyendo a $12.5 \mathrm{mg}$ durante tres semanas, $10 \mathrm{mg}$ durante cuatro a seis semanas, seguido de una reducción de $1 \mathrm{mg}$ (4-8 semanas), o bien la reducción en días alternos (por ejemplo, 10/7.5 mg en días alternos y así sucesivamente). No se recomienda el uso de $\operatorname{AINE}(33,34)$.

\section{Osteoporosis}

Se caracteriza por una disminución de masa ósea, asociada a mayor riesgo de fractura, especialmente en cadera, vértebra y muñeca. Pese a que la edad es un determinante de osteoporosis, en el anciano es pobremente evalúa, sin tratamiento oportuno. La mayor prevalencia de fracturas se aumenta por el riesgo de caídas, las enfermedades concomitantes y comedicamentos. La suplencia con calcio y vitamina $\mathrm{D}$ en la dieta reduce el riesgo de fractura, la deficiencia de vitamina $\mathrm{D}$ en el anciano se ha asociado con mayor riesgo de caídas dado por inestabilidad y disminución en la fuerza muscular. Se ha demostrado reducción de riesgo de fractura con la administración con terapia antirresortiva en pacientes mayores de 75 años con osteoporosis. El ácido zoledrónico ha demostrado reducción del riesgo de fracturas en pacientes frágiles con fractura de cadera reciente. Para teriparatide faltan estudios en pacientes adultos mayores, en los subgrupos de pacientes mayores de 75 años no hay diferencia significativas, el ranelato de estroncio es el primer fármaco con eficacia documentada contra la fractura no vertebrales y de fracturas vertebrales, con una eficacia sostenida de más de cinco años. Es de vital importancia la prevención de caídas con cualquier estrategia para disminuir el riesgo de fracturas en la vejez. En la actualidad, la osteoporosis senil es subdiagnosticada y el tratamiento inadecuado, la edad no debería ser un obstáculo para el diagnóstico y la intervención de esta patología (35-37).

\section{Manejo del dolor}

Dado las múltiples comorbilidades y los dilemas éticos al momento de evaluar algunos fármacos, las estrategias de manejo del dolor en los ancianos no han sido evaluadas de modo sistemático y la mayoría se basa en la experiencia clínica en pacientes más jóvenes o con dolor oncológico. La poca evidencia demuestra que el uso de enfoques no farmacológicos, en combinación con fármacos analgésicos, puede resultar en mejor manejo del dolor, con menos dependencia a medicamentos (38). Dentro de las estrategias no farmacológicas debemos considerar: la terapia física, terapia ocupacional orientada a garantizar independencia, estimulación nerviosa eléctrica transcutánea, educación, acupuntura y apoyo social. El simple hecho de contar con un ambiente de apoyo mejora el control del dolor. La educación brindada al paciente ayuda a superar ciertas barreras, como la resistencia a expresar el dolor, el temor a la adicción, o la idea de que la medicación debe ser reservada para cuando el dolor sea mayor (39).

\section{Consideraciones en farmacocinética y farmacodinamia}

En el anciano la mayoría de los procesos fisiológicos se ven alterados, ejemplo, la absorción no se altera con la edad, pero puede tener un retardo debido a la disminución de la perfusión intestinal. La distribución de los fármacos cambia, al cambiar con la edad la composición corporal, la pérdida de la masa muscular y del agua corporal total, con aumento de la grasa. Los fármacos hidrosolubles tienen un menor volumen de distribución, con concentraciones más elevadas y los fármacos liposolubles se distribuyen ampliamente, ocasionando una eliminación retardada.

La metabolización por el sistema del citocromo P450 no se altera con la edad. La disminución de la masa hepática puede aumentar la concentración de la sustancia activa de aquellos fármacos con un gran metabolismo de primer paso, y prolongar la vida media sérica de los fármacos metabolizados por el hígado. La eliminación renal de muchos fármacos disminuye tanto con la edad como con determinadas enfermedades. Los analgésicos narcóticos suelen tener un efecto farmacodinámico aumentado, por lo que es necesario disminuir la dosis, e igualmente las múltiples patologías y polifarmacia en los ancianos dan un mayor riesgo de presentar interacciones farmacológicas $(40,41)$.

Los fármacos que tienen unión alta a proteínas pueden tener alteraciones al ser administrados en conjunto con AINES o metotrexate, ejemplo; disminuye los efectos de la warfarina y las sulfonilúreas y aumentan los niveles séricos de digoxina, sulfonamidas, penicilinas, fenitoína y ácido valproico.

Los efectos gastrointestinales de los AINES son la principal causa de morbilidad entre pacientes ancianos, con alta tasa de hemorragia gastrointestinal, los AINES afectan la protección de la mucosa gástrica mediada por las prostaglandinas, por lo cual la adición de un inhibidor de bomba de protones y erradicación de la infección por H. pylori, se recomienda en conjunto al uso de estos medicamentos. Igualmente altera el flujo sanguíneo renal y la filtración glomerular, se asocia a descompensación de insuficiencia cardiaca congestiva, nefrosis, cirrosis o hipovolemia, llevando a retención de agua y sodio, lo que da lugar a edema periférico e hipertensión arterial. Dosis altas de estos medicamentos, principalmente indometacina y la fenilbutazona pueden causar cefalea, confusión, alteraciones cognitivas, depresión o paranoia en ancianos, por lo que siempre que sea posible deben evitarse. De los efectos a favor de estos 
medicamentos se ha asociado con reducción del riesgo de pólipos colónicos y cáncer colorrectal, y con un menor riesgo de enfermedad de Alzheimer.

Los analgésicos narcóticos pueden causar sedación excesiva y trastornos cognitivos en ancianos. Sin embargo, la tolerancia y la dependencia a estos fármacos no son menos frecuentes entre ancianos que en los jóvenes. En los ancianos, pueden ocasionar estreñimiento grave o impactación fecal, se recomienda una dieta rica en fibra. Al combinarse con fármacos depresores del sistema nervioso central, como los sedantes hipnóticos, tranquilizantes mayores, antidepresivos tricíclicos y el alcohol, producen sedación excesiva, trastornos cognitivos e incluso coma.

Los analgésicos opioides pueden ser útiles para controlar el dolor en ancianos cuidadosamente seleccionados. Las guías de uso de opioides a largo plazo incluyen:

- Elegir pacientes con una fuente de dolor estructuralmente bien definida.

- No incluir personas con síndromes musculoesqueléticos inespecíficos.

- Realizar una evaluación psicosocial previa.

- Tratar de evitar su uso en pacientes con antecedentes de adicciones.

- Realizar evaluaciones regulares de la eficacia del tratamiento.

- Si el tratamiento no es efectivo, se lo debe suspender.

El tramadol, usado para el tratamiento del dolor moderado a levemente intenso, puede constituir una alternativa para la osteoartritis que no calma con acetaminofen; se trata de un analgésico de acción central más seguro para personas con problemas renales o gastrointestinales (constipación), con bajo potencial para dependencia.

Los opiáceos de acción prolongada, como la metadona, deben ser utilizados con gran precaución, dado que la droga puede acumularse con toxicidad retardada. Las dosis de inicio y las posteriores deben ser inferiores en los ancianos debido a factores farmacocinéticos y farmacodinámicos relacionados con la edad.

Debe evitarse los antidepresivos tricíclicos si hay hipotensión ortostática preexistente, así como lipotimias, glaucoma, boca seca, estreñimiento o retención urinaria a través de sus efectos anticolinérgicos. En caso de estar indicado un antidepresivo tricíclico deben preferirse los que tienen menos actividad anticolinérgica. Por alteraciones en conducción cardiaca y riesgo de arritmias, deberían utilizarse con precaución en pacientes con enfermedad cardiaca. Los antidepresivos tricíclicos han sido aplicados para el tratamiento del dolor neuropático crónico, pero debería considerarse preferiblemente otras drogas como gabapentina, pregabalina, carbamacepina, fenitoína y ácido valproico.

Los inhibidores de la recaptación de serotonina pueden causar el síndrome de secreción inadecuada de hormona antidiurética y se han asociado con un mayor riesgo de caídas en ancianos, por lo que deben evitarse.
Los corticoides son medicamentos seguros y de mucha utilidad en el anciano, puede empeorar la hipertensión y falla cardiaca por retención de sodio, la diabetes mellitus, la osteoporosis, la debilidad muscular y el glaucoma, predisponer a infecciones en los pacientes ancianos (el riesgo de infección se relaciona con la dosis y la duración del tratamiento). Debe tenerse en cuenta que los ancianos son más susceptibles con alteraciones del eje hipotálamo-hipófisis-suprarrenal, por lo que son necesarios un descenso lento y gradual y una monitorización estrecha durante la reducción de la dosis. Los glucocorticoides también presentan efectos adversos a nivel del sistema nervioso central, como depresión, manía, delirio, psicosis y trastornos cognitivos en el anciano. Debe evaluarse la posibilidad de la miopatía por esteroides.

\section{Fármacos modificadores de la enfermedad y agentes biológicos}

En general el uso de la terapia DMAR no está contraindicada en el anciano, debe considérese algunos puntos especiales, por ejemplo el uso de hidroxicloroquina y cloroquina debe evitarse en enfermedad previa de la retina. $\mathrm{La}$ insuficiencia renal requiere reducir la dosis de metotrexate, con el fin de evitar la toxicidad farmacológica, principalmente citopenia, además puede desencadenar brotes de herpes zoster por lo cual debe suspenderse ante esta entidad. La ciclosporina puede empeorar la hipertensión, causar insuficiencia renal, está contraindicado en los pacientes con insuficiencia renal preexistente.

El infliximab se ha asociado con un empeoramiento de la insuficiencia cardiaca congestiva y fallecimiento por esta causa, por lo que su uso está contraindicado en pacientes con insuficiencia cardiaca grados III o IV de la clasificación de la New York Heart Association. Aún no se conoce claramente si el etanercept tiene efectos similares, por lo que éste debe usarse con precaución.

Las interacciones más importantes son entre azatioprina y alopurinol, y entre metotrexate y trimetoprima, que pueden causar toxicidad grave de la médula ósea. Aunque existe interacción farmacocinética entre metotrexate y aspirina y otros AINES, que resulta en el aumento de la biodisponibilidad del metotrexate, el uso simultáneo de estos fármacos no se ha asociado con toxicidad clínicamente importante (42-44).

\section{Referencias}

1. Bruckenthal P. Assessment of Pain in the Elderly Adult. Clin Geriatr Med 2008; 24: 213-36.

2. Hadjistavropoulos T, Herr K, Turk DC, Fine PG, Dworkin RH, Helme R, et al. An interdisciplinary expert consensus statement on assessment of pain in older persons. Clin J Pain 2007; 23: 1-43.

3. Bosley BN, Weiner DK, Rudy TE, Granieri E. Is chronic nonmalignant pain associated with decreased appetite in older adults? Preliminary evidence. $J$ Am Geriatr Soc 2004; 52: 247-51.

4. Gallagher R, Verma S, Mossey J. Chronic pain: sources of late-life pain and risk factors for disability. Geriatrics 2000; 55: 40-7.

5. AGS Panel on Persistent Pain in Older Persons. The management of persistent pain in older persons . J Am Geriatr Soc 2002; 50: 205-24.

6. Closs S, Briggs M. Patients' verbal descriptions of pain and discomfort following orthopaedic surgery. Int J Nurs Stud 2002; 39: 563-72. 
7. Wynne C, Ling S, Remsburg R. Comparison of pain assessment instruments in cognitively intact and cognitively impaired nursing home residents. Geriatr Nurs 2000; 21: 20-3.

8. Charette S.Bruce F. Rheumatic Diseases in the Elderly: Assessing Chronic Pain. Rheum Dis Clin N Am 2007; 33:109-22

9. Biumstein G, Gorevic P. Rheumatologic illnesses Treatment strategies for older adults. Geriatrics 2005; 60: 28-35.

10. Herr K, Garand L. Assessment and measurement of pain in older adults. Clin Geriatr Med 2001; 17: 457-78.

11. Biumstein G, Gorevic P. Rheumatologic illnesses Treatment strategies for older adults. Geriatrics 2005; 60: 28-35.

12. Broder J. Snarski J. Back Pain in the Elderly. Clin Geriatr Med 2007; 23: 271-89

13. Wolf A y Pfleger B. Burden of major musculoskeletal conditions. Bulletin of the World Health Organization 2003; 81: 646-56.

14. Loeser R. Aging and the etiopathogenesis and treatment of osteoarthritis. Rheum Dis Clin North Am 2000; 26: 547-67.

15. Murray C, Lopez A. The global burden of disease. A comprehensive assessment of mortality and disability from diseases, injuries and risk factors in 1990 and projected to 2020. Cambridge. Harvard School of Public Health on behalf of the WHO and the World Bank, 1996.

16. Cardiel R. Riedemann P. Epidemiología de las Enfermedades Reumáticas. En Introducción a la Reumatologia, Tercera Edición. Editor Martínez- Elizondo P. Fondo Editorial CMR, 2003.

17. Yazici Y, Paget S. Elderly-Onset Rheumatoid Arthritis. Rheum Dis Clin N Am 2000; 26: 517-26.

18. Tutuncu Z, Kavanaugh A. Rheumatic Disease in the Elderly: Rheumatoid Arthritis. Rheum Dis Clin N Am 2007; 33: 57-70.

19. Riise T, Jacobsen B, Gran J. Incidence and prevalence of rheumatoid arthritis in the county of Troms, northern Norway. J Rheumatol 2000; 27: 1386-9.

20. Huusko T, Korpela M, Karppi P, Avikainen V, Kautiainen H, Sulkava R. Threefold increased risk of hip fractures with rheumatoid arthritis in Central Finland. Ann Rheum Dis 2001; 60: 521-2.

21. Canoso J, Alvarez-Nemegyei J. Soft Tissue Rheumatology. En: Lahita RG, Weinstein A, editores. Educational Review Manual in Rheumatology. 3.a ed. New York: Castle Connolly Graduate Medical; 2006.

22. Gudmundsson K, Arngrimsson R, Sigfusson N, Bjornsson A, Jonsson T. Epidemiology of Dupuytren's disease: clinical, serological, and social assessment. The Reykjavik Study. J Clin Epidemiol 2000; 53: 291-6.

23. Wolfe F, Ross K, Anderson J, Russell IJ, Hebert L. The prevalence and characteristics of fibromyalgia in the general population. Arthritis Rheum 1995; 38:19-28.

24. Canoso J, Álvarez J. Reumatismo de partes blandas. En: Alarcon-Segovia D, Molina J, editores. Tratado Hispanoamericano de Reumatología. 1 a ed. Bogotá: Nomos; 2006.

25. Yunus M, Holt G, Masi A, Aldag J. Fibromyalgia syndrome among the elderly. Comparison with younger patients. J Am Geriatr Soc 1998; 11: 987-95.
26. Wallace K, Riedel A, Joseph-Ridge N, Wortmann R. Increasing prevalence of gout and hyperuricemia over 10 years among older adults in a managed care population. J Rheumatol 2004; 31: 1582-7.

27. Pascual E, Sivera F. Therapeutic advances in gout. Curr Opin Rheumatol 2007; 19: $122-7$.

28. Wise C. Crystal-Associated Arthritis in the Elderly. Rheum Dis Clin N Am 207; 33: $33-55$.

29. Restrepo J. Pascual E. Gota en el anciano. Rev Colomb Reumatol 2008: 15: 55-8.

30. Kammer G y Mishra N. Systemic Lupus Erythematosus in the Elderly. Rheum Dis Clin N Am 2000; 26: 475-92.

31. Sánchez A. Lupus eritematoso sistémico. Aspectos clínicos poco frecuentes. Reumatol Clin. 2008; 4: 28-30.

32. Petursdottir V, Johansson H, Nordborg E, Nordborg C. The epidemiology of biopsy-positive giant cell arteritis: special reference to cyclic fluctuations. Rheumatology 999; 38: 1208-12.

33. Dasgupta B, Matteson E, Maradit-Kremers H. Management guidelines and outcome measures in polymyalgia rheumatica. Clin Exp Rheumatol 2007; 25: $130-6$.

34. Salvarani C, Macchioni P, Boiardi L. Polymyalgia rheumatica. Lancet 1997; 350: $43-7$.

35. Boonen S, Dejaeger E, Vanderschueren D, Venken K, Bogaerts A, Verschueren $\mathbf{S}$, et al. Osteoporosis and osteoporotic fracture occurrence and prevention in the elderly: a geriatric perspective. Best Pract Res Clin Endocrinol Metab 2008; 2: 765-85.

36. Van Staa T, Dennison E, Leufkens HG, Cooper C. Epidemiology of fractures in England and Wales. Bone 2001; 29: 517-22.

37. Lindsay R, Silverman S, Cooper C, Hanley DA, Barton I, Broy SB, Licata A et al. Risk of new vertebral fracture in the year following a fracture. JAMA 2001; 285: $320-3$.

38. Deane G, Smith H. Overview of Pain Management in Older Persons. Clin Geriatr Med 2008; 24: 185-201.

39. Ruoff G. Challenges of Managing Chronic Pain in the Elderly. Semin Arthritis Rheum 2002; 32: 43-50.

40. Barkin L, Barkin S, Barkin D. Pharmacotherapeutic Management of Pain with a Focus Directed at the Geriatric Patient. Rheum Dis Clin N Am 2007; 33: 1-31.

41. Strassels S, McNicol E, Suleman R. Pharmacotherapy of Pain in Older Adults. Clin Geriatr Med 2008; 24: 275-98.

42. Diaz-Borjon A, Weyand,C, Goronzy J. Treatment of chronic inflammatory diseases with biologic agents: opportunities and risks for the elderly. Exp Gerontol 2006; 41: 1250-5.

43. Ranganath V, Furst D. Disease-Modifying Antirheumatic Drug Use in the Elderly Rheumatoid Arthritis Patient. Rheum Dis Clin N Am 2007; 33: 197-217.

44. Fam A. Gout in the elderly. Clinical presentation and treatment. Drugs Aging 1998; 13: 229-43. 